\title{
e-Phaïstos
}

e-Phaïstos

Revue d'histoire des techniques / Journal of the history

of technology

II-1 | 2013

Savoirs et sciences sur les machines

\section{Des formes à consommer. Pensées et pratiques du design industriel en France (1945-1980)}

Shapes to Consume. Theories and Practices of Industrial Design in France

(1945-1980)

\section{Claire Leymonerie}

\section{(2) OpenEdition}

Journals

Édition électronique

URL : http://journals.openedition.org/ephaistos/1353

DOI : $10.4000 /$ ephaistos. 1353

ISSN : 2552-0741

Éditeur

IHMC - Institut d'histoire moderne et contemporaine (UMR 8066)

Édition imprimée

Date de publication : 1 juin 2013

Pagination : 105-110

ISSN : 2262-7340

Référence électronique

Claire Leymonerie, «Des formes à consommer. Pensées et pratiques du design industriel en France (1945-1980) », e-Phaïstos [En ligne], II-1 | 2013, mis en ligne le 15 décembre 2018, consulté le 15 septembre 2020. URL : http://journals.openedition.org/ephaistos/1353 


\title{
Claire Leymonerie
}

\section{Des formes à consommer. Pensées et pratiques du design industriel en France (1945-1980)}

\author{
Thèse de doctorat \\ École des hautes études en sciences sociales (EHESS), Paris \\ Centre de recherches historiques $(\mathrm{CRH})$ \\ Soutenue le 14 décembre 2010 \\ 1 vol. (633 p.), bibliographie, annexes
}

Directeurs de thèse :

Patrick FRIDENSON, directeur d'études, EHESS

Franck COCHOY, professeur, Université Toulouse 2 Le Mirail

Jury :

Marie-Emmanuelle CHESSEL, directrice de recherche au CNRS, CRH, EHESS (présidente)

Jean-Pierre DAVIET, professeur émérite, Université de Caen (rapporteur)

Danièle VOLDMAN, directrice de recherche au CNRS, CHS, Université Paris 1 Panthéon-Sorbonne (rapporteur)

Armand HATCHUEL, professeur, école des Mines ParisTech
Avant de démarrer cette présentation, je souhaite vous remercier d'avoir accepté de participer à ce jury et d'avoir manifesté ainsi de l'intérêt pour mon travail. J'en profite également pour remercier le public venu assister à cette soutenance.

La thèse que je présente aujourd'hui, pour l'introduire en quelques mots, porte sur l'histoire du design industriel en France des années 1940 aux années 1970. Ce choix peut étonner: en effet, le design industriel n'existe pas, du moins pas sous ce nom, jusqu'au milieu des années 1960. C'est donc aussi d'esthétique industrielle, de stylisme industriel, de formes utiles, de création industrielle dont il est question dans mon travail. Autant de vocables par lesquels ce que nous nommons aujourd'hui le design industriel émerge et se construit.

Le point de départ de cette thèse a été l'exploration, dans le cadre de mon DEA, des archives du Salon des arts ménagers. À travers ces archives, le salon m'est apparu comme un microcosme permettant de visualiser l'ensemble d'un secteur économique - celui de l'équipement ménager - et de saisir les dynamiques qui animent ses différents acteurs. J'ai pu observer les stratégies de concurrence entre fabricants, qui se jouent à travers la différenciation de leurs produits et de leurs prix, mais aussi à travers l'aménagement de 
leurs stands et l'éloquence de leurs démonstrateurs. J'ai pu saisir les rapports entre distributeurs et fabricants, et le salon m'est apparu enfin comme le théâtre d'un apprentissage de la consommation de masse pour les visiteurs.

J'ai souhaité, dans ma thèse, maintenir cette approche à l'échelle d'un secteur économique, en l'occurrence celui des petits appareils électroménagers. Je me suis donc dirigée vers une histoire des entreprises du secteur, et je suis partie en quête d'archives : j'ai parcouru le très riche fonds Moulinex déposé aux Archives départementales du Calvados à la suite du dépôt de bilan de l'entreprise en 2000, j'ai consulté au Conservatoire SEB les archives de l'entreprise Calor. La presse professionnelle a constitué une ressource d'une très grande richesse pour retracer l'histoire du marché des appareils électroménagers, dont elle est un acteur à part entière: je pense ici à des revues comme La Quincaillerie moderne ou L'Argus ménager.

Mais surtout, et pour en venir à ce qui constitue au terme de ce parcours le cour de ma thèse, les archives du Salon des arts ménagers m'ont permis de découvrir l'action de l'association Formes Utiles. Avant de devenir une association indépendante, Formes Utiles est née en 1949 dans le giron de l'Union des artistes modernes, c'est-à-dire dans la continuité du mouvement moderne constitué dans l'Entre-deux-guerres dans les domaines de l'architecture et des arts appliqués. Constitué à l'occasion de la présentation d'une exposition en 1949 au Musée des arts décoratifs, le groupe Formes Utiles s'est consacré par la suite à la réalisation de sélections thématiques au sein des produits présentés au Salon des Arts ménagers et à leur présentation sous forme d'expositions. L'étude de Formes Utiles et de son action de promotion des formes industrielles m'a ouvert une première fenêtre sur l'histoire du design industriel français, dont j'ai élargi ma connaissance au contact de Catherine Geel, avec qui j'ai collaboré pour la préparation de l'exposition consacrée au designer Pierre Paulin à la Villa Noailles en 2007.

J'ai poursuivi cette exploration du design industriel français en mobilisant des sources variées. Les archives Calor au Conservatoire SEB m'ont ainsi réservé une heureuse surprise: un dossier retraçant de manière détaillée la collaboration nouée, dans les années 1950, entre l'entreprise et le bureau d'études Technès spécialisé en esthétique industrielle. Cependant, les traces de l'activité des designers restent rares dans les archives d'entreprises industrielles. Je suis donc allée voir du côté des designers eux-mêmes et de leurs bureaux d'études: j'ai réalisé de nombreux entretiens, j'ai classé les archives des designers Yves Savinel et Gilles Rozé, qui ont établi une longue collaboration avec SEB des années 1970 aux années 1990. Toujours pour ce qui concerne les sources, je souhaite insister de nouveau sur la richesse des revues, en l'occurrence Esthétique industrielle, puis Créé, qui sont à la fois des témoins et des acteurs de la vie professionnelle du design industriel. Enfin, j'ai consulté les archives des institutions publiques chargées de la promotion du design industriel dans les années 1970 : le Centre de création industrielle, que j'appellerai dans la suite de cet exposé le CCI, le Conseil supérieur de la création esthétique industrielle.

Cette exploration a été l'occasion de déceler le silence de l'historiographie française sur le design en général et sur le design français en particulier. Les rares études consacrées en France à l'histoire du design sont marquées par le poids écrasant des modèles étrangers, et notamment de l'Allemagne et de l'Italie. La France pose pourtant des problèmes spécifiques et pertinents pour l'histoire du design. L'enjeu majeur qui se dessine est celui de la rupture avec un modèle des arts décoratifs lié aux savoirfaire et aux modes de production artisanaux, et dont les produits sont destinés à une clientèle élitiste et fortunée. Le prestige des arts décoratifs en France constitue une explication à l'émergence 
tardive du design industriel. Il contribue également à expliquer la cécité relative de l'historiographie sur le sujet.

Je dois souligner cependant l'apport de plusieurs historiennes américaines (Leora Auslander, Deborah Silvermann, Susan Tise, Nancy Troy), l'apport également de l'historien Stéphane Laurent pour situer ce modèle des arts décoratifs dans un contexte économique et politique, et pour expliquer les raisons et les ressorts de sa permanence. La recherche pionnière de Jocelyne Le Bœuf permet de saisir les ressorts de l'émergence du design industriel en France à travers le parcours de Jacques Viénot, fondateur de l'Institut d'esthétique industrielle. Ces lectures m'ont permis de dégager une conviction: l'émergence du design industriel n'est pas tant une affaire de tendance esthétique que de division du travail. En effet, le design industriel, en tant qu'activité, ne peut exister qu'à partir du moment où sont délimités, au sein du processus de production industrielle des objets, un espace, des compétences et une profession spécifiquement dédiés à leur mise en forme.

Ce questionnement premier est venu s'enrichir au fil de la thèse de multiples thématiques, dont je souhaite maintenant faire un rapide bilan. La thèse fait ressortir tout d'abord la pertinence de la notion de génération pour interpréter la chronologie de l'histoire du design. Notre première partie est consacrée à l'étude de deux organisations de promotion du design industriel dans les années 1950, Formes Utiles d'une part, l'Institut d'esthétique industrielle d'autre part. Elle fait apparaitre clairement que les débats intellectuels, les réseaux sociaux mobilisés constituent l'aboutissement d'une dynamique amorcée dès les années 1920 et ralentie par la crise économique et la guerre. En revanche, il se produit dans le courant des années 1960 un véritable changement de décor : on voit entrer en scène une nouvelle génération, on voit la mobilisation de nouveaux paradigmes théoriques et l'on lit en creux l'influence des évènements de mai 1968.

La chronologie du design industriel français est également marquée par un moment de cristallisation au tournant des années 1970: de nombreux bureaux d'études sont alors en activité, une nouvelle revue apparaît en 1969 - la revue Créé -, de nouvelles institutions de formation et de promotion sont constituées, notamment le CCI. Se tiennent également des expositions marquantes : «Qu'est-ce que le design ? " en 1969, «Le Design français » en 1971. Cependant, cette cristallisation est de courte durée et s'érode très rapidement au fil de la décennie : Créé s'interrompt en 1977, le CCI est intégré dans la logique culturelle et patrimoniale du Centre Pompidou, le rythme de création des bureaux d'études se ralentit, la crise économique et la saturation des marchés modifient le rôle économique du design. La fin de la décennie 1970 clôt donc en demi-teinte ce que l'on peut interpréter rétrospectivement comme un âge d'or du design industriel français.

À mes yeux, le résultat le plus frappant de la thèse est que le design industriel n'émerge pas là où on l'attend. La France se distingue en effet nettement d'un modèle dominant qui établit la filiation entre mouvement moderne et design industriel : en Allemagne, par exemple, le lien est direct entre les expériences fondatrices du Bauhaus dans l'Entre-deux-guerres et la pratique du design industriel au sein de la Hochschule für Gestaltung d'Ulm à partir des années 1950. Dans le cas de la France, le mouvement moderne, incarné dans les domaines des arts décoratifs et des arts appliqués par l'Union des artistes modernes, aboutit à une relative impasse en dépit des innovations formelles indéniables réalisées par ses membres. Cette impasse s'explique d'une part par l'incapacité à concilier activité de création et enjeux marchands, d'autre part par l'incapacité à concevoir une division du travail créatif et à donner naissance à une figure professionnelle de spécialiste des formes industrielles. Ni les décorateurs, ni les architectes 
présents au sein du mouvement moderne ne s'aventurent réellement dans la conception de formes pour les produits de l'industrie.

C'est donc autour de l'Institut d'esthétique industrielle que se cristallise une pensée, une pratique et un milieu professionnel de l'esthétique industrielle en France. Le trajet de Jacques Viénot, fondateur de l'Institut, depuis la galerie DIM qui produit du mobilier luxueux de style Art Déco pour une clientèle très fortunée, jusqu'au bureau d'études Technès, l'un des premiers bureaux d'esthétique industrielle français, marque une transition improbable entre arts décoratifs et design industriel. Une filiation évidente m'est également apparue entre l'Institut d'esthétique industrielle et le milieu des promoteurs de la rationalisation des méthodes de production dans les entreprises industrielles, dont la naissance dans l'Entre-deuxguerres a été relatée par l'historienne Aimée Moutet. On retrouve le terreau intellectuel commun du taylorisme et des stratégies d'action similaires pour convaincre les dirigeants d'entreprise. Enfin, le modèle professionnel du styliste industriel, défini au sein de l'Institut d'esthétique industrielle, est calqué sur celui de l'ingénieur-conseil et vient donc grossir les rangs des spécialistes du conseil aux entreprises.

La conduite de cette recherche m'a convaincue, est c'est le second résultat de la thèse, de la nécessité de prendre en compte une typologie des objets techniques pour comprendre la spécificité du design industriel. La conception formelle d'un élément de mobilier, table, chaise, fauteuil, ne requiert par les mêmes compétences que la conception d'un objet intégrant une dimension technologique, aussi modeste soit-elle (résistance ou moteur électrique pour ce qui concerne les appareils électroménagers). Ces objets possèdent une architecture spécifique, faite de la juxtaposition d'un dispositif technique qui en constitue le cour et d'une enveloppe, carrosserie ou carter. De fait, au sein du groupe professionnel des designers, ce ne sont pas les mêmes qui conçoivent du mobilier d'une part, et des objets à fort contenu technique d'autre part. C'est ce qui ressort notamment de l'analyse statistique que j'ai réalisée à partir de l'annuaire des concepteurs édité par le CCI en 1975.

Le troisième résultat que j'aimerais souligner est la mise en évidence d'un processus de professionnalisation particulier pour le design industriel. La constitution d'un groupe professionnel autour du design industriel passe bien sûr par des stratégies classiques telles que la création de formations spécialisées ou d'organes syndicaux. On est forcé pourtant de constater la faiblesse de ce processus d'institutionnalisation et de souligner l'incapacité de la profession à mettre à profit les quelques rares moyens de promotion mis à disposition par l'État. En témoigne l'échec du Conseil supérieur de la création esthétique industrielle: créé en 1971 dans le cadre de la politique d'encouragement à l'innovation mise en œuvre sous la présidence Pompidou, il disparaît dès 1975 .

Les designers ont donc recours à d'autres stratégies pour se constituer et se faire reconnaître comme profession. Le travail sémantique y tient une place importante : ceux que nous appelons par commodité ici les designers mènent une réflexion sans cesse recommencée sur la bonne manière de se nommer et de se définir. Cette stratégie passe également par la mise en scène de ce que l'on pourrait appeler des épreuves de professionnalisation. C'est le cas par exemple des bancs d'essai organisés par l'Institut d'esthétique industrielle, séances au cours desquelles des industriels sont invités à soumettre leurs productions à l'avis de spécialistes de l'esthétique industrielle, qui leur prodiguent des conseils pour l'amélioration des formes. À travers les bancs d'essai, l'esthétique industrielle se définit, en situation, comme profession de conseil aux entreprises industrielles. Enfin, la stratégie de professionnalisation passe par la mobilisation 
d'instruments de légitimation d'ordre méthodologique : dans les années 1960 et 1970, les designers mettent en avant le product planning et les méthodologies de création pour promouvoir leur activité dans les entreprises.

Quatrième résultat important à mes yeux, la thèse permet de définir la position du design entre marché et organisation. Elle permet de retracer le déplacement progressif du design industriel depuis l'espace marchand, où il se fait connaître et reconnaître par les acteurs économiques - on pense ici à l'action de Formes Utiles au Salon des arts ménagers - vers une structure organisationnelle, le bureau d'études, lui permettant d'engager des collaborations avec les entreprises. Cependant, même au terme de ce parcours, le design continue de prétendre jouer un rôle nodal dans le fonctionnement du marché, en fournissant une assistance aux consommateurs et en leur proposant des dispositifs de jugement pour effectuer leurs choix. C'est ce dont témoigne, pour les années 1970, l'élaboration et l'éphémère mise en ouvre, au sein du CCI, du système d'information sur les produits : ce système mobilisait les ressources de l'informatique pour répertorier l'ensemble des produits présents sur le marché français et proposer à l'utilisateur des critères de tri, telles que les fonctions remplies par l'appareil, sa taille, son prix ou sa marque, afin de sélectionner les produits correspondant le mieux à ses besoins et l'aider à effectuer son choix.

Enfin, le dernier résultat sur lequel j'aimerais insister est l'importance de la réflexion théorique qui accompagne l'essor du design industriel en France. Cette réflexion se mène d'abord, dans les années 1950, au sein des groupes qui assurent la promotion du design industriel. L'architecte André Hermant, théoricien des « formes utiles », construit sur le socle du fonctionnalisme une pensée inquiète de l'industrialisation ; a contrario, le philosophe Étienne Souriau et l'ingénieur Georges Crombet, théoriciens de l'esthétique industrielle, puisent dans le taylorisme afin de définir une activité de création compatible avec les conditions de la production industrielle. Dans les années 1960 et 1970, le design, plus largement les objets et la société de consommation, sont au centre de débats qui animent les milieux universitaires et intellectuels. Prolongeant le projet de sémiologie générale formulé par Roland Barthes, Jean Baudrillard procède à une relecture du concept marxiste de " fétichisme de la marchandise » pour dénoncer les fondements théoriques du design: fonction et besoin ne seraient que des alibis dissimulant l'inscription des objets dans un mécanisme de différenciation sociale, bien loin de l'idéal démocratique du mouvement moderne. Face à lui, le physicien et philosophe Abraham Moles, qui assure le passage vers la France des théories de la Hochschule für Gestaltung d'Ulm, réaffirme la dimension essentiellement fonctionnelle des objets au dépend de leur dimension signifiante. Il appelle à l'avènement d'un "néo-fonctionnalisme » où le designer jouerait le rôle de médiateur aménageant les rapports de l'homme à la société via son environnement matériel, selon un schéma inspiré de la cybernétique. Ces réflexions théoriques prennent à partie les designers, et ceux-ci les prennent en compte pour définir leur profession et adopter un positionnement éthique quant à leur rôle dans le système de production et de consommation des biens.

Au fil de cette thèse j'ai suivi la piste du design industriel à partir de l'étude du secteur du petit électroménager. Ce parti pris induit nécessairement une vision partielle du sujet, appelle des études complémentaires et me conduit donc à dégager quelques pistes de recherches à explorer. À l'occasion de l'exposition «Design français » organisée en 1971 par le CCI se dégage un profil spécifique du design en France, caractérisé par l'importance des secteurs de l'équipement lourd: transport, mobilier urbain, aménagement des 
espaces industriels, machines-outils, engins de travaux publics. De plus, en me concentrant sur la sphère marchande, je n'ai abordé qu'à la marge une histoire de l'enseignement du design industriel qui promet pourtant d'être très féconde. Il faudrait ainsi retracer l'histoire de la formation en design de produit à l'ENSAD, la naissance d'un enseignement du design à destination des ingénieurs au sein de l'Université de Technologie de Compiègne, mais aussi la place du design dans l'éphémère existence de l'Institut de l'environnement au début des années 1970. 